\title{
Combining Bioimpedance and Myographic Signals for the Assessment of COPD during Loaded Breathing
}

\author{
Dolores Blanco-Almazán, Willemijn Groenendaal, Manuel Lozano-García, Luis Estrada-Petrocelli, Senior \\ Member, IEEE, Lien Lijnen, Christophe Smeets, David Ruttens, Francky Catthoor, Fellow, IEEE, and \\ Raimon Jané, Senior Member, IEEE
}

\begin{abstract}
Chronic Obstructive Pulmonary Disease (COPD) is one of the most common chronic conditions. The current assessment of COPD requires a maximal maneuver during a spirometry test to quantify airflow limitations of patients. Other less invasive measurements such as thoracic bioimpedance and myographic signals have been studied as an alternative to classical methods as they provide information about respiration. Particularly, strong correlations have been shown between thoracic bioimpedance and respiratory volume. The main objective of this study is to investigate bioimpedance and its combination with myographic parameters in COPD patients to assess the applicability in respiratory disease monitoring. We measured bioimpedance, surface electromyography and surface mechanomyography in forty-three COPD patients during an incremental inspiratory threshold loading protocol. We introduced
\end{abstract}

This work was supported in part by the Universities and Research Secretariat from the Ministry of Business and Knowledge/Generalitat de Catalunya under Grant FI-DGR and Grant GRC 2017 SGR 01770, in part by the Agencia Estatal de Investigación from the Spanish Ministry of Science, Innovation and Universities and the European Regional Development Fund, under the Grant RTI2018 098472-B-I00, and in part by the CERCA Programme/Generalitat de Catalunya.

D. Blanco-Almazán, M. Lozano-García, L. Estrada-Petrocelli and R. Jané are with the Institute for Bioengineering of Catalonia (IBEC), The Barcelona Institute of Science and Technology, 08028 Barcelona Spain and also the Biomedical Research Networking Center in Bioengineering, Biomaterials and Nanomedicine (CIBERBBN), 08028, Barcelona, Spain (e-mail: dblanco@ibecbarcelona.eu, mlozano@ibecbarcelona.eu, lestrada@ibecbarcelona.eu and rjane@ibecbarcelona.eu).

D. Blanco-Almazán, M. Lozano-García, and R. Jané are also with the Department of Automatic Control (ESAll) at the Universitat Politècnica de Catalunya, 08034 Barcelona, Spain.

L. Estrada-Petrocelli is also with the Facultad de Ingeniería at the Universidad Latina de Panamá, 0823-00933 Panama City, Republic of Panama.

W. Groenendaal is with imec the Netherlands/Holst Centre, Eindhoven 5656AE, the Netherlands (email: Willemijn.Groenendaal@imec.nl).

L. Lijnen was with Faculty of Medicine and Life Sciences at Hasselt University, 3500 Hasselt, Belgium (e-mail: lien.lijnen@hotmail.com).

C. Smeets is with the Future Health Department at Ziekenhuis OostLimburg, 3600 Genk, Belgium (e-mail: Christophe.Smeets@zol.be).

D. Ruttens is with the Department of Respiratory Medicine at Ziekenhuis Oost-Limburg, 3600 Genk, Belgium. (e-mail: David.Ruttens@zol.be).

F. Catthoor is with imec and KU Leuven, Heverlee 3001, Belgium (email: Francky.Catthoor@imec.be). two novel features that can be used to assess COPD condition derived from the variation of bioimpedance and the electrical and mechanical activity during each respiratory cycle. These features demonstrate significant differences between mild and severe patients, indicating a lower inspiratory contribution of the inspiratory muscles to global respiratory ventilation in the severest COPD patients. In conclusion, the combination of bioimpedance and myographic signals provides useful indices to noninvasively assess the breathing of COPD patients.

Index Terms-bioimpedance, chronic obstructive pulmonary disease, inspiratory threshold protocol, myographic signals, wearables

\section{INTRODUCTION}

C HRONIC Obstructive Pulmonary Disease (COPD) is a common disease in the adult population being a major cause of chronic morbidity and mortality. COPD is the fifth leading cause of death worldwide representing an important economic and social burden worldwide [1], [2]. The current diagnosis and the assessment of COPD severity require a spirometry test, which measures the pulmonary function by a maximal maneuver to quantify the level of airflow limitation [3]-[5]. However, this test does not provide a complete assessment of the complex clinical condition of the COPD patients. Additional information sources, such as questionnaires are used to complement the diagnosis and severity assessment [4]. Consequently, COPD assessment results in a not portable procedure which requires subjective information. Improving the current method is a challenge for the current health systems worldwide.

More comfortable and less invasive methods have been studied as alternative to classical methods to monitor COPD and other pulmonary diseases. These novel techniques such as thoracic bioimpedance [6]-[8], electromyography [9], [10] or, inductance plethysmography [11] have been shown to be able to provide useful respiratory information, however, there is still a lack of evidence of their usefulness in healthcare applications [12].

Thoracic bioimpedance is a noninvasive method that measures the change in impedance of that zone over time. All 
tissues, organs and fluids of the thoracic zone contribute to changes in thoracic bioimpedance. However, previous studies have shown a clear linear relation between thoracic bioimpedance and respiratory volume [6]-[8], [11], [13]. On the other hand, clinical evidence is still limited, only a few studies have investigated and showed the applicability of the use of bioimpedance in respiratory diseases, focusing on infants suffering from reduced lung function [14]-[16]. Other noninvasive measurements such as surface electromyography (sEMG) and surface mechanomyography (sMMG) have been investigated for assessment of muscle function in healthy and diseased state [9], [10], [17]-[22]. These studies showed strong correlations between sEMG and sMMG and classical invasive measurements of respiratory muscle function [17]-[19]. Consequently, both bioimpedance and myographic signals are potential alternatives to the traditional invasive and obtrusive methods in characterizing respiratory diseases.

COPD patients present airflow limitation [4], [23] and commonly, respiratory muscle dysfunction [24]-[26] which contribute to the sensation of breathlessness. These respiratory conditions could be targeted with the combination of bioimpedance and sEMG and sMMG. Both bioimpedance and myographic recordings are noninvasive and comfortable techniques that allow ambulatory measurements.

The aim of the present study is the evaluation of the combination of bioimpedance and myographic parameters in COPD patients and assess the applicability for respiratory monitoring. This is the first study in combining bioimpedance and myographic parameters as noninvasive indices for COPD assessment. Positive results in this study will reinforce the combination of bioimpedance and myographic signals for monitoring in pulmonary diseases like COPD.

\section{Materials and Methods}

\section{A. Subjects}

Fifty COPD patients were recruited at Ziekenhuis OostLimburg (Genk, Belgium), during their consultation or rehabilitation session. All the patients were diagnosed with COPD before the recruitment in this study.

The study was approved by the local institutional medical ethics committee from Ziekenhuis Oost-Limburg with reference 18/0047U. The study followed the World Medical Association's Declaration of Helsinki on Ethical Principles for Medical Research Involving Humans Subjects. All patients provided written consent before they participated in the study.

\section{B. Respiratory protocol}

The study consisted of a spirometry test and an incremental inspiratory threshold loading protocol. The novelty of the presented study is the simultaneous measurement of bioimpedance and myographic signals in COPD patients to investigate ventilatory responses during inspiratory loading. From the spirometry test, we obtained the forced vital capacity (FVC) and forced expiratory volume in one second $\left(\mathrm{FEV}_{1}\right)$ which are parameters used to classify the severity of COPD [4]. The ventilatory response during the inspiratory threshold

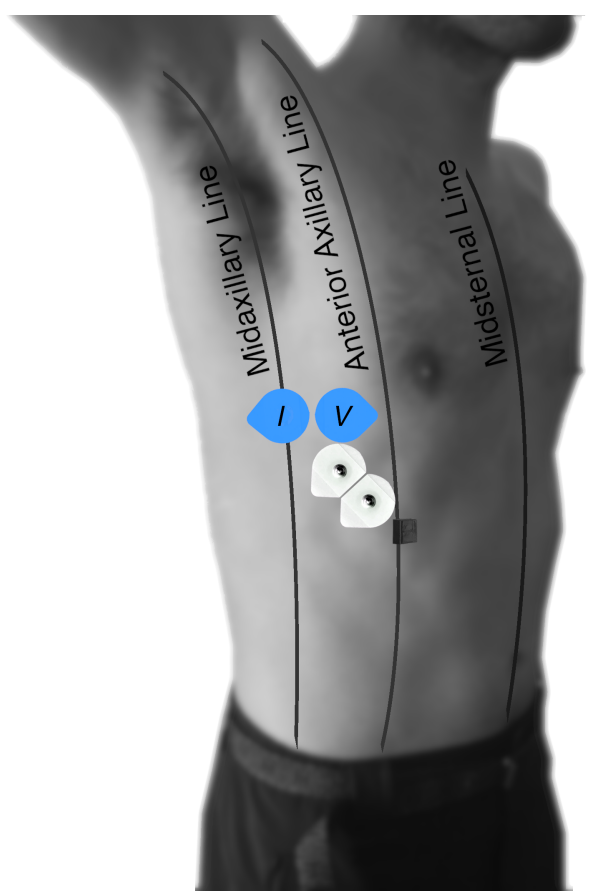

Fig. 1. Location of the electrodes and accelerometer. The electrode configuration for bioimpedance measurement was from the midsternal line. Only the right side is represented (colored electrodes). I denotes the injecting current electrode and $V$ the voltage measurement ones. The electrodes for sEMG measurements and the accelerometer were placed on the seventh or eighth intercostal space on the right side of the thorax (white electrodes and black box) .

loading protocol was measured by the bioimpedance and myographic signals. Applying inspiratory loads require the patient to increase the overall inspiratory effort needed to breath. Inspiratory loading protocols have been used previously in studies of inspiratory muscle function [17], [18], [27], [28].

In the protocol, first the patient's maximal static inspiratory pressure (MIP) was determined by a maximal volitional maneuver [29]. Next, the inspiratory loads were progressively increased proportionally to the patient's MIP. The protocol started with two minutes of quiet breathing (no inspiratory load) and was followed by loaded breathing using five different inspiratory loads, specifically $12 \%, 24 \%, 36 \%, 48 \%$ and 60 $\%$ of the patients' MIP. The patients were free to adapt their breathing to overcome the loads. Each load consisted of 30 breaths and there was a two-minute resting period in between all loads.

The patients were comfortably seated in an upright position and they wore a disposable nose clip to block nasal breathing for the entire protocol.

\section{Data acquisition}

The physiological signals were acquired using two different systems, a wearable research prototype device (Stichting imec the Netherlands, Eindhoven, the Netherlands) and a standard wired acquisition system (MP150, Biopac Systems, Inc., Goleta, CA, USA).

Thoracic bioimpedance (bioZ) was acquired by the wearable device using a tetrapolar electrode configuration $(\mathrm{Ag} / \mathrm{AgCl}$ 
TABLE I

DEMOGRAPHIC AND ANTHROPOMETRIC DATA FOR COPD PATIENTS

\begin{tabular}{|c|c|c|c|c|}
\hline & $\begin{array}{l}\text { Mild } \\
\mathrm{n}=5\end{array}$ & $\begin{array}{c}\text { Moderate } \\
n=16\end{array}$ & $\begin{array}{l}\text { Severe } \\
\mathrm{n}=22\end{array}$ & $\mathrm{p}$-value \\
\hline Male (Female) & $4(1)$ & $9(7)$ & $20(2)$ & \\
\hline Age yr & $68.00(63.75-72.75)$ & $65.00(60.00-69.00)$ & $65.50(61.00-70.00)$ & 0.56 \\
\hline BMI kg/m² & $25.05(23.32-28.53)$ & $24.09(23.30-28.59)$ & $25.42(21.43-28.72)$ & 0.87 \\
\hline MIP $\mathrm{cm}^{2} \mathrm{H}_{2} \mathrm{O}$ & $51.00(30.00-78.25)$ & $43.50(27.00-66.00)$ & $55.00(47.00-65.00)$ & 0.52 \\
\hline FVC $\%$ pred & $116.60(105.13-122.00)^{*}$ & $87.75(78.30-95.55)$ & $79.80(59.40-100.20)^{*}$ & 0.01 \\
\hline $\mathrm{FEV}_{1} \%$ pred & $93.50(90.93-103.77)^{*}$ & $58.45(54.85-69.05) \#$ & $42.45(34.50-45.00)^{* \#}$ & $\mathrm{p}<0.001$ \\
\hline $\mathrm{FEV}_{1} / \mathrm{FVC} \%$ & $67.20(64.00-69.44)^{*}$ & $51.66(46.03-61.55) \#$ & $37.84(33.34-42.11)^{* \#}$ & $\mathrm{p}<0.001$ \\
\hline
\end{tabular}

The data are presented as median (first - third quartile) values. BMI: body mass index; MIP: maximum inspiratory pressure; FVC: forced vital capacity; $\mathrm{FEV}_{1}$ : forced expiratory volume in one second. The p-values were obtained from Kruskal-Wallis' test. The * and \# symbols denote the groups with significant differences, p-value $<0.05$.

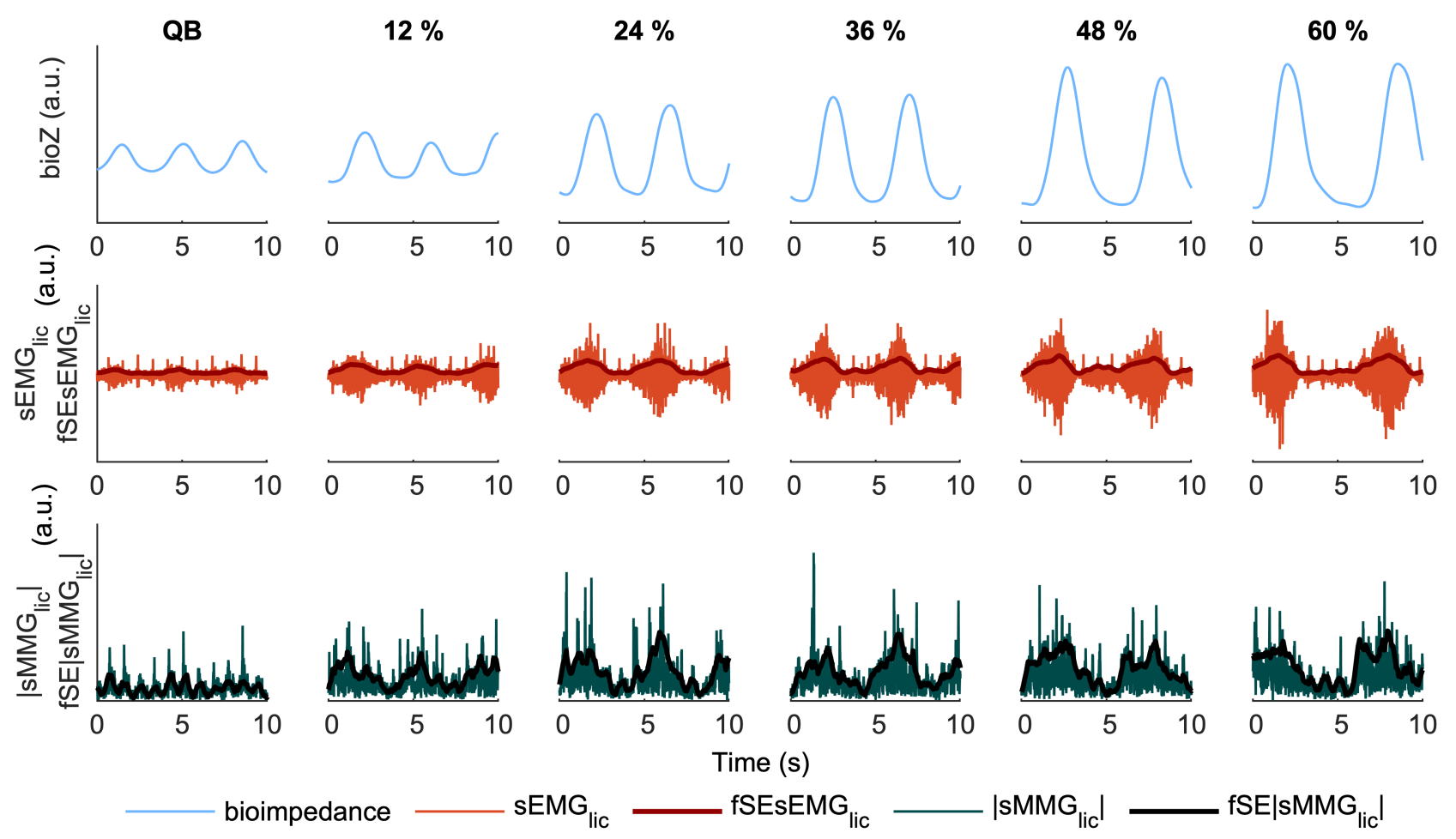

Fig. 2. Temporal representation of the bioimpedance, $\boldsymbol{s} \boldsymbol{E} \boldsymbol{M} \boldsymbol{G}_{l i c}$ and $\left|\boldsymbol{s} \boldsymbol{M} \boldsymbol{M} \boldsymbol{G}_{l i c}\right|$ preprocessed signals for a patient. In addition to the preprocessed myographic signals, $\boldsymbol{f S E s E} \boldsymbol{M} \boldsymbol{G}_{l i c}$ and $\boldsymbol{f} \boldsymbol{S E | s} \boldsymbol{M} \boldsymbol{M} \boldsymbol{G}_{l i c} \mid$ are shown as these signals' amplitude estimations. Ten seconds of each part of the inspiratory protocol is shown to exhibit the changes in the signals due to the imposed inspiratory loads.

electrodes, Kendall H92SG, Covidien Inc., MA, USA) symmetrical from the midsternal line as Fig. 1 shows. The two injection current electrodes were placed on the mid-axillary lines at approximately $7 \mathrm{~cm}$ from the end of the axilla. The voltage measurement electrodes were horizontally separated $5 \mathrm{~cm}$ from the injecting ones to the midsternal line. The injection current parameters were selected based on previous studies [7], [8] and the capabilities of the device. The injection current amplitude was $110 \mu \mathrm{App}$ at a frequency of $80 \mathrm{kHz}$. The presented setup gave a good sensitivity and linearity of bioimpedance signal, as well as a good signal-to-noise ratio as Fig. 2 shows.

Surface electromyography from the lower intercostal spaces $\left(\mathrm{sEMG}_{l i c}\right)$ was measured using two disposable $\mathrm{Ag} / \mathrm{AgCl}$ electrodes (Kendall H92SG, Covidien Inc.) connected to a differential amplifier (EMG100C, Biopac Systems, Inc.). The electrodes were placed between the right mid-axillary line and the anterior axillary line and along the seventh or eighth intercostal spaces [10], [30]. The $\mathrm{sEMG}_{l i c}$ was amplified 1000 times and band-pass filtered using an analog low-pass filter $\left(f_{c}\right.$ $=5 \mathrm{kHz})$ and an analog high-pass filter $\left(f_{c}=1 \mathrm{~Hz}\right)$.

Surface mechanomyography, also from the lower intercostal spaces, $\left(\mathrm{sMMG}_{l i c}\right)$ was acquired with a tri-axial accelerometer (TSD109C2, Biopac Systems, Inc.) connected to its corresponding interface (HLT100C, Biopac Systems, Inc.). The accelerometer was placed on the patient's skin with adhesive rings close to the $\mathrm{sEMG}_{l i c}$ electrodes. In particular, the accelerometer was placed near the patient's right anterior axillar line and over the seventh or eighth intercostal space [18], [20] (Fig. 1).

Respiratory airflow was also acquired by the Biopac system using an airflow transducer (pneumotach transducer TSD107B, 
Biopac Systems,Inc.). The airflow transducer was connected to a differential amplifier (DA100C, Biopac Systems, Inc.) which amplified 1000 times the airflow signal and low-pass filtered it $\left(f_{c}=300 \mathrm{~Hz}\right)$, analogically. The patients breathed through a disposable mouthpiece with a bacterial filter (AFT36, Biopac Systems, Inc.) wearing a disposable nose clip.

The data collection included the electrocardiogram (ECG) measurements from both devices for synchronization purposes.

The signals acquired by Biopac system were digitized with a sampling rate of $10 \mathrm{kHz}$. The bioimpedance and the ECG signals from the wearable device were acquired at 16 and 512 $\mathrm{Hz}$ respectively. These settings were used in our previous study [8] where we confirmed that $16 \mathrm{~Hz}$ sampling was sufficient to capture respiratory information.

The spirometry test was performed with MasterScreen CPX Metabolic Cart (JAEGER, Würzburg, Germany). An inspiratory muscle trainer device (POWERbreathe KH2, POWERbreathe International Ltd, Southam, UK) was used for the MIP maneuver and to impose inspiratory threshold loads.

\section{Data Analysis}

Pre-processing: The ECG signals were used to align the recordings of the two measurement systems in time. For that, we computed the lag that maximizes the cross-correlation of the ECG signals and subsequently we used it to align the signals in time.

Thoracic bioimpedance was resampled to $200 \mathrm{~Hz}$ by cubic interpolation to increase the time resolution. Given that the respiratory information is in a low-frequency band, the bioZ signal was band-pass filtered by a high-pass filter (zero-phase $4^{\text {th }}$ order Butterworth, $f_{c}=0.05 \mathrm{~Hz}$ ) and a low-pass filter (zero-phase $4^{\text {th }}$ order Butterworth, $f_{c}=1 \mathrm{~Hz}$ ).

$s E M G_{l i c}$ signals were low-pass filtered to avoid aliasing $\left(8^{\text {th }}\right.$ order Chebyshev Type I, $f_{c}=800 \mathrm{~Hz}$ ) before the resampling. $s E M G_{l i c}$ signals were decimated to $2 \mathrm{kHz}$, highpass filtered (zero-phase $8^{\text {th }}$ order Butterworth, $\mathrm{f}_{c}=10 \mathrm{~Hz}$ ) and low-pass filtered (zero-phase $8^{\text {th }}$ order Butterworth, $\mathrm{f}_{c}$ $=600 \mathrm{~Hz}$ ). $s M M G_{l i c}$ signals consisted of three signals from the three accelerometer orthogonal axes. The $s M M G_{l i c}$ signals were first low-pass filtered to avoid aliasing $\left(8^{\text {th }}\right.$ order Chebyshev Type $\mathrm{I}, \mathrm{f}_{c}=80 \mathrm{~Hz}$ ). Then, the $s M M G_{l i c}$ signals were resampled to $200 \mathrm{~Hz}$ and band-pass filtered by a highpass filter (zero-phase $8^{\text {th }}$ order Butterworth, $\mathrm{f}_{c}=5 \mathrm{~Hz}$ ) and a low-pass filter (zero-phase $8^{\text {th }}$ order Butterworth, $\mathrm{f}_{c}=40$ $\mathrm{Hz})$. The global acceleration $\left|s M M G_{l i c}\right|$ was obtained as the Euclidian norm of the acceleration in each axis [31].

The airflow signal was decimated to $200 \mathrm{~Hz}$ after being antialiasing filtered (Chebyshev Type I, $\mathrm{f}_{c}=80 \mathrm{~Hz}$ ). The airflow signal was filtered in the frequency band from 0.05 to $1 \mathrm{~Hz}$. The same filters were applied to the airflow signals as to the bioZ signals.

Fixed Sample Entropy Analysis of myographic signals: The myographic signals, $s E M G_{l i c}$ and $\left|s M M G_{l i c}\right|$, were analyzed by the fixed sample entropy technique (fSampEn). fSampEn measures the complexity and self-similarity of time-series data [32]. The particularity of fSampEn is that the sample entropy is not computed for the entire signal, but it is computed
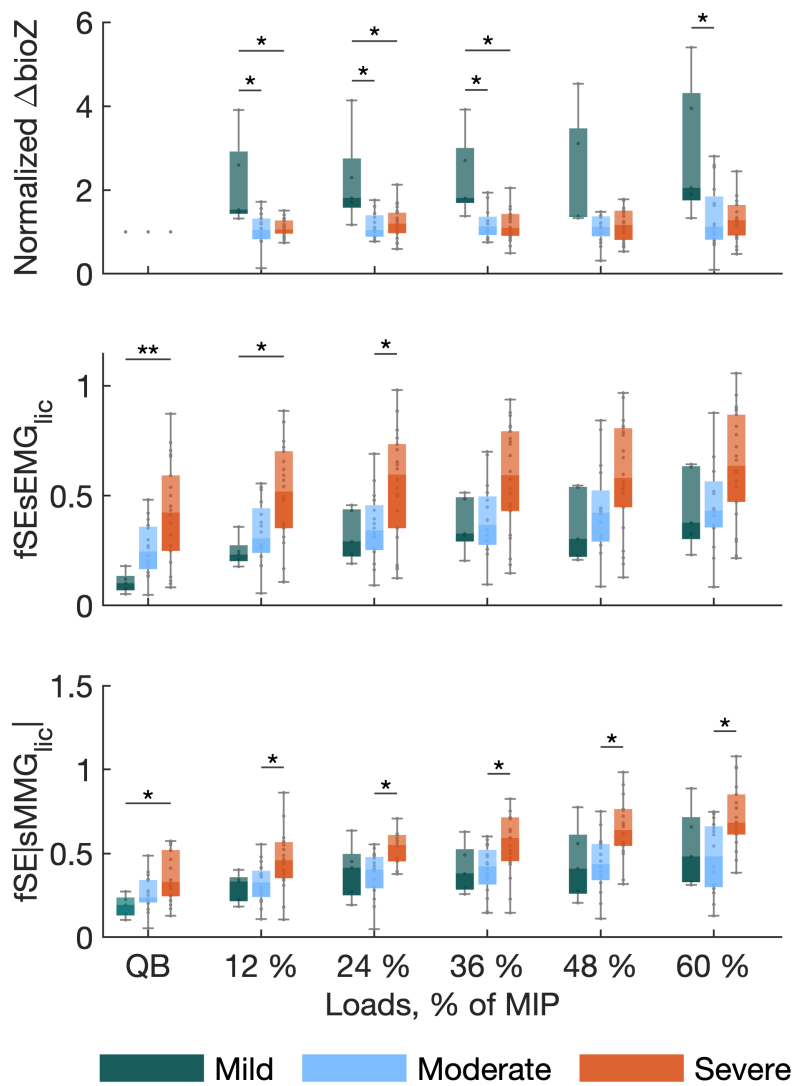

Fig. 3. Evolution of the data parameters values from bioZ, $f \boldsymbol{S E s E} M G_{l i c}$ and $\boldsymbol{f} \boldsymbol{S E | s M M G _ { l i c } |}$ of the three severity groups with increasing inspiratory loads. The values are shown as box plots, showing the median, first and third quantiles, and, the maximum and minimum values. The statistical analysis was performed to evaluate significant differences between groups by Kruskal-Wallis' test. The pairwise $p$-values were corrected using the Bonferroni's correction. * denotes $p$ value $<0.05$ and $^{* *}$ denotes $p$-value $<0.01$.

in moving windows using the same parameters. Fixing the parameters allows fSampEn to track the amplitude of the signal as well as the complexity [33]. Recently fSampEn has being used in myographic signals because it is less sensitive to deterministic components like the cardiac interference [10], [33].

The selection of fSampEn parameters is important when assessing respiratory effort using myographic signals [34]. Consequently, we selected the fSampEn parameters following the conclusions of the Lozano-Garcia et al. [31] study where different combinations of fSampEn parameters were evaluated in terms of their ability to estimate inspiratory muscle activity. We used windows lengths of $500 \mathrm{~ms}$ with a $90 \%$ of overlap, an embedded dimension of 2 and a tolerance of 0.2 for $s E M G_{l i c}$ and 0.5 for $\left|s M M G_{l i c}\right|$. Thus, we obtained an estimated amplitude signal from each myographic signal, denoted as $f S E s E M G_{l i c}$ and $f S E\left|s M M G_{l i c}\right|$, as shown in Fig 2. Using the same parameters for fSampEn calculations for all myographic signals allowed us to quantitatively compare sample entropy values between patients. 
Data indices: We calculated common parameters related to breathing pattern, such as inspiratory time, duty cycle (inspiratory time over respiratory time), respiratory frequency and inspiratory volume. We used these parameters to characterize changes in breathing patterns with increasing inspiratory loads.

Our main objective was to evaluate the ventilatory response in COPD patients of different severities during inspiratory loading using noninvasive parameters from bioZ, $f S E s E M G_{l i c}$ and $f S E\left|s M M G_{l i c}\right|$. Parameters were calculated cycle-by-cycle, hereto, signals were segmented in respiratory cycles by a thresholding algorithm applied to the airflow signal [35].

For the thoracic bioimpedance signal we computed the amplitude, defined as the peak-to-peak difference of bioZ $(\Delta b i o Z)$, during each respiratory cycle. Previous studies showed the linear relationship between bioimpedance and respiratory volume [7], [13] even during inspiratory loaded breathing [8]. Therefore, the variation in amplitude of the bioimpedance signal for each respiratory cycle was related to patients' respiratory volume changes. Absolute bioimpedance values are dependent on the patients' anthropometry, meaning the absolute changes in amplitude cannot be compared between different patients. To be able to compare the changes in bioimpedance between patients, we normalized each patient's $\triangle b i o Z$ parameter values by the median of this parameter obtained during quiet breathing. Therefore, the $\Delta b i o Z$ values are dependent on the baseline conditions of each patient and the values represent their variation related to quiet breathing.

On the other hand, we obtained the mean values of the $f S E s E M G_{l i c}$ and $f S E\left|s M M G_{l i c}\right|$ signals during each inspiratory phase to quantify the level of muscle activity. These myographic measures were previously suggested as inspiratory muscle force estimation [18]. Additionally, we computed two ratios using these two parameters and $\Delta b i o Z$. In particular, we computed the ratio between bioimpedance amplitude and $f S E s E M G_{l i c}, \mathrm{BEr}$, and the ratio between bioimpedance amplitude and $f S E\left|s M M G_{l i c}\right|$, BMr:

$$
\begin{gathered}
B E r_{i}=\frac{\Delta b i o Z^{i}}{f S E s E M G_{l i c}^{i}} \\
B M r_{i}=\frac{\Delta b i o Z^{i}}{f S E\left|s M M G_{l i c}\right|^{i}}
\end{gathered}
$$

where $i$ is $\mathrm{i}^{\text {th }}$ respiratory cycle used for the calculation of each parameter. These ratios represent the contribution of inspiratory muscles to bioimpedance during the breathing.

We selected ten cycles for each patient and load based on the similarity of the cycles in terms of breathing pattern, resulting sixty cycles per subject. The breathing pattern parameters used for the selection were inspiratory time, duty cycle, respiratory frequency and also $\Delta b i o Z$. Firstly, the median values from these four parameters were computed and then the ten cycles which parameters were closest to the medians were automatically selected. The aim of this selection was to obtain stable cycles in terms of breathing pattern to get representative values for each subject and load.

In summary, we computed three indices from bioZ, $f S E s E M G_{l i c}$ and $f S E\left|s M M G_{l i c}\right|$ signals, and, two novel ratios between these parameters to evaluate the respiration activity in COPD patients during loaded breathing.

\section{E. Statistical Analysis}

The statistical analysis was designed to evaluate the breathing evolution of the three COPD severity groups. Consequently, we computed a representative value for each patient and load for all parameters and ratios, thus we obtained six values of each parameter and patient. The representative values were calculated as the median of the parameters from the ten selected cycles.

We performed a paired Wilcoxon's signed rank test to compare the representative breathing parameters in quiet breathing and the highest load (60\% of patient's MIP) for each patients' group individually. In addition, a Kruskal-Wallis' test was performed to evaluate the differences of the parameters and ratios between the three COPD groups. If the Kruskal-Wallis' test resulted in a p-value less than 0.05 , we evaluated pairwise the parameters with Bonferroni's p-value correction.

\section{REsults}

The study included fifty COPD patients, 38 males and 12 females. Seven patients were excluded due to allergic reaction (1), technical device problems (4) and wrong sensor attachment (2).

The patients were classified in three groups, depending on their $\mathrm{FEV}_{1}$ [4]. The three groups were: mild $\left(\mathrm{FEV}_{1} \geqslant 80\right.$ $\%)$, moderate $\left(50 \% \leqslant \mathrm{FEV}_{1}<80 \%\right)$ and severe $\left(\mathrm{FEV}_{1}\right.$ $<50 \%$ ). Table I shows the distribution of the patients in the severity groups and their demographic and anthropometric data. Statistical differences were analyzed by the KruskalWallis' test. We did not find significant differences in age, BMI and MIP between the groups. Significant differences were found as expected in pulmonary function, $\mathrm{FVC} \%$ pred, $\mathrm{FEV}_{1}$ $\%$ pred and $\mathrm{FEV}_{1} / \mathrm{FVC} \%$.

Forty-three COPD patients performed an incremental inspiratory threshold loading protocol while physiological signals were recorded. During the respiratory protocol the patients were free to adapt their breathing to overcome the loads. Meaning, the breathing pattern could change from quiet breathing to the highest load ( $60 \%$ of patients' MIP). Four breathing pattern parameters were computed and statistically analyzed during quiet breathing and the $60 \%$ load using the paired Wilcoxon signed rank test. In particular, inspiratory volume, inspiratory time, duty cycle and respiratory frequency values are shown in Table II for the three severity groups. For the moderate and severe groups, we found statistical differences in inspiratory time and duty cycle. No significant differences were found in inspiratory volume and respiratory frequency values. These results show that inspiratory loading modified the breathing pattern of the three groups but in a different way depending on the severity.

Bioimpedance, $s E M G_{l i c}$ and $\left|s M M G_{l i c}\right|$ were measured during the respiratory protocol. Fig. 2 shows the evolution of these signals with load, as well as the amplitude estimation of the myographic signals, fSEsEMG $G_{l i c}$ and fSE|sMMG $\left|G_{l i c}\right|$, obtained from the fSampEn method [33]. 
TABLE II

BREATHING PATTERN PARAMETERS OF COPD PATIENTS DURING QUIET BREATHING AND $60 \%$ OF MAXIMUM INSPIRATORY PRESSURE

\begin{tabular}{|c|c|c|c|c|c|c|}
\hline & \multicolumn{2}{|c|}{$\begin{array}{l}\text { Mild } \\
\mathrm{n}=5\end{array}$} & \multicolumn{2}{|c|}{$\begin{array}{c}\text { Moderate } \\
\mathrm{n}=16\end{array}$} & \multicolumn{2}{|c|}{$\begin{array}{l}\text { Severe } \\
\mathrm{n}=22\end{array}$} \\
\hline & QB & $60 \%$ & QB & $60 \%$ & QB & $60 \%$ \\
\hline $\mathrm{V}_{I} \mathrm{~L}$ & $0.9(0.8-1.1)$ & $1.6(0.9-2.9)$ & $1.1(0.9-1.2)$ & $1.1(0.9-1.3)$ & $1.1(0.9-1.3)$ & $0.9(0.7-1.5)$ \\
\hline $\mathrm{t}_{I} \mathrm{~s}$ & $1.6(1.3-1.7)$ & $1.6(1.6-1.9)$ & $1.4(1.1-1.5)$ & $1.6(1.2-1.8)^{*}$ & $1.2(0.9-1.6)$ & $1.3(1.1-1.9)^{*}$ \\
\hline $\mathrm{t}_{I} / \mathrm{t}_{T O T}$ & $0.44(0.40-0.44)$ & $0.39(0.38-0.50)$ & $0.38(0.36-0.42)$ & $0.47(0.38-0.51)^{*}$ & $0.35(0.32-0.39)$ & $0.39(0.34-0.41)^{*}$ \\
\hline $\mathrm{f}_{R} \min ^{-1}$ & $18.2(15.3-18.8)$ & $14.2(12.4-18.5)$ & $17.6(15.4-21.0)$ & $17.0(15.1-19.8)$ & $17.4(13.5-21.3)$ & $17.2(12.8-20.3)$ \\
\hline
\end{tabular}
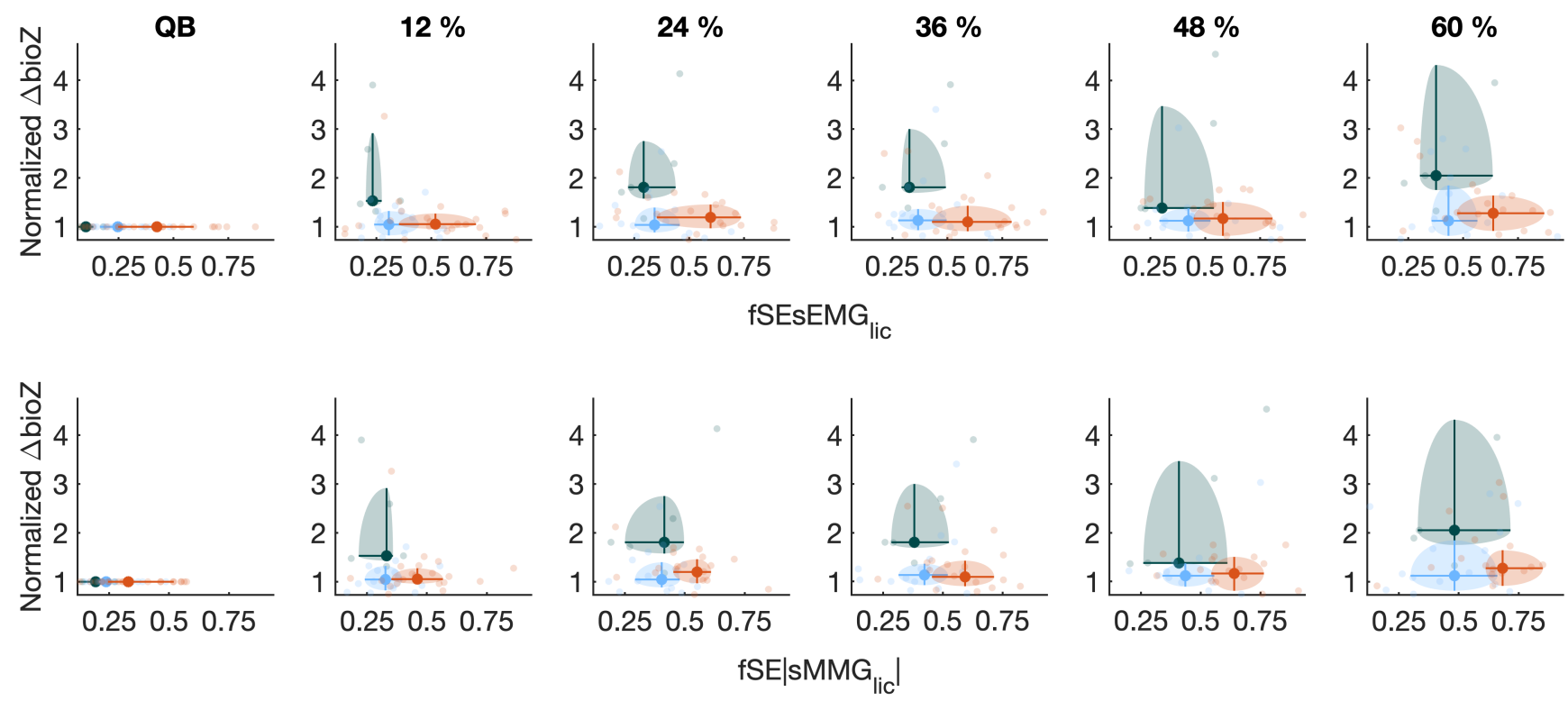

\section{fSE|SMMG}

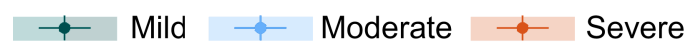

Fig. 4. Comparison between the bioZ, $\boldsymbol{f S E s E} \boldsymbol{M} \boldsymbol{G}_{l i c}$ and $\boldsymbol{f} \boldsymbol{S E}\left|\boldsymbol{s} \boldsymbol{M} \boldsymbol{M} \boldsymbol{G}_{l i c}\right|$ parameters of the three severity groups over the increasing inspiratory loads. The markers show the median values of the parameters and the bars represent the first and third quantile. The highlighted zones represent the interquartile range of the parameters.

From bioZ, $f S E s E M G_{l i c}$ and $f S E\left|s M M G_{l i c}\right|$ we computed three parameters for each respiratory cycle. The parameter values are shown in Fig. 3 grouped by the COPD severity levels mild, moderate and severe. Note that $\triangle$ bio $Z$ values were normalized by the value during quiet breathing, i.e., the values from the loaded breathing represent the changes from the baseline. $\triangle b i o Z$ evolved differently for the severity groups, exhibited by the significant differences, p-value $<$ 0.05 . On the other hand, for the myographic parameters, the median values increased in the loaded breathing with respect to quiet breathing for all severity groups. Furthermore, the severe group exhibited higher myographic median values than the two other groups. Significant differences were observed between the mild and severe groups during quiet breathing. However, the significant differences between the moderate and severe group were more frequent and occurred also during the loaded breathing. Note that for $\left|s M M G_{l i c}\right|$ parameters the significance between these two groups was observed for all the respiratory protocol parts.
Bioimpedance and myographic parameters were studied in relation to each other for the different loads and COPD severity levels, Fig. 4. This representation, which combines bioimpedance and myographic information, exposes further differences between COPD severity groups. The most separated groups in this analysis for all the loads are the mild and severe groups. In Fig. 4, the differences in quiet breathing cannot be observed so easily because of the $\Delta$ bioZ $Z$ normalization which used the quiet breathing values to scale the loaded breathing values. These severity differences during quiet breathing and also the differences during loaded breathing are quantified using novel ratio, $\mathrm{BEr}$ and $\mathrm{BMr}$, as shown in Fig. 5. Higher ratio values were obtained for the mild COPD group in both ratios. For the moderate and severe groups, the ratio values were lower than for the mild group, being the medians of the $\mathrm{BEr}$ and $\mathrm{BMr}$ of the severe group always the lowest. We found significant differences between both $\mathrm{BEr}$ and $\mathrm{BMr}$ of the mild and severe groups for all the parts of the protocol. Consequently, the most extreme COPD severity 
groups differed statistically in $\mathrm{BEr}$ and $\mathrm{BMr}$ for quiet and loaded breathing indicating higher efficiency in bioimpedance variation over myographic activity for the mild group than for the severe group.

\section{DISCUSSION}

The main objective of the study was to investigate bioimpedance and its combination with myographic parameters in COPD patients during inspiratory loading to assess the applicability in respiratory disease monitoring. For the first time, bioimpedance parameters and its relation to myographic parameters were evaluated as noninvasive indices for the assessment of COPD patients. In particular, we computed parameters from bioimpedance, $s E M G_{l i c}$, and, $\left|s M M G_{l i c}\right|$ in COPD patients during an incremental threshold inspiratory loading protocol. From these data parameters we computed two novel ratios between bioimpedance and the myographic parameters, BEr and BMr. The ratios represent the relationship of inspiratory muscle activity with global respiratory ventilation. We found significant differences in the $\mathrm{BEr}$ and $\mathrm{BMr}$ ratios between mild and severe COPD patients. These differences indicate lower inspiratory contribution of inspiratory muscles to ventilation in severe COPD patients than in the mild COPD patients.

Thoracic bioimpedance is a noninvasive technique that measures the impedance changes of the body tissues, organs and fluids in the thoracic zone. Several studies confirmed the linear relationship between respiratory volume and thoracic bioimpedance in healthy subjects during normal and loaded breathing [6]-[8], [11], [13]. Although other contributions are reflected in the bioimpedance signal, the respiratory volume is the main contributor [36]. Only few studies evaluated the use of bioimpedance in respiratory disease monitoring or diagnosis, specifically thoracic bioimpedance was evaluated in infants with reduced lung function [14]-[16]. These studies confirmed the applicability of bioimpedance for noninvasive monitoring in this respiratory condition. Consequently, bioimpedance has been suggested as a noninvasive technique to measure respiration. Furthermore, because of the linear relation, bioZ can be used as an estimate for respiratory volume.

Inspiratory threshold loading protocols are associated with changes in breathing pattern and, particularly, with an increase of tidal volume with respect to baseline in healthy subjects [22], [27], [28], [37]. The increase in $\Delta$ bioZ can only be observed in the mild group of which the median values were higher than median values of the other two groups (Fig. 3). On the other hand, the median value of $\Delta$ bioZ of the moderate and severe groups remained unchanged, showing almost no variation. This difference in behavior in the moderate and severe groups could be caused by airflow limitation which is known to occur in COPD patients [4], [23]. Note that we normalized $\Delta b i o Z$ values by the value during quiet breathing, therefore we obtained the changes with respect to baseline. Additionally, the airflow limitation was also confirmed by inspiratory volumes values obtained by spirometry in Table II. Unlike in healthy subjects, we did not find significant differences in inspiratory volumes between quiet breathing and
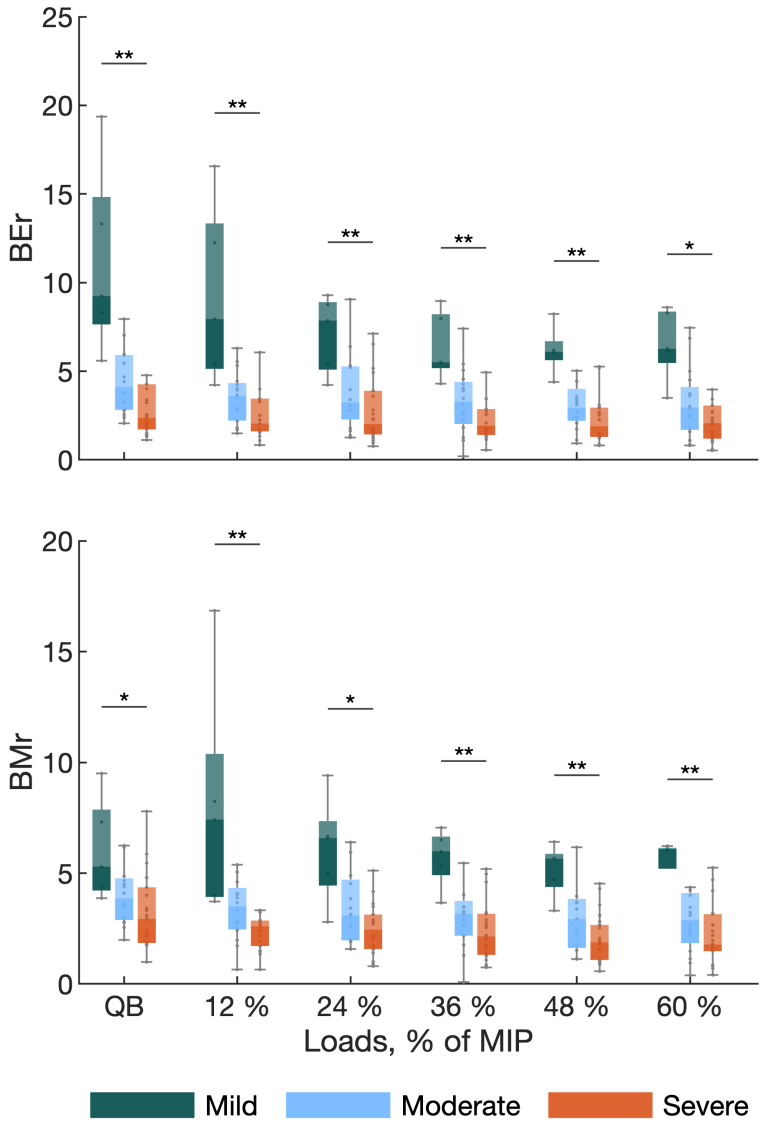

Fig. 5. Ratios computed from bioZ, $\boldsymbol{f} \boldsymbol{S} \boldsymbol{E} \boldsymbol{s} \boldsymbol{E} \boldsymbol{M} \boldsymbol{G}_{l i c}$ and $f \boldsymbol{S E}\left|s \boldsymbol{M} M G_{l i c}\right|$ of the three severity groups over the inspiratory threshold loading protocol. The values are drawn as box plots, showing the median, first and third quantiles, and, the maximum and minimum values. The statistical analysis was performed to evaluate significant differences between groups by Kruskal-Wallis' test. The pairwise $p$ values were corrected by the Bonferroni's correction. * denotes $p$-value $<0.05$ and $^{* *}$ denotes $p$-value $<0.01$.

the highest load. As expected, the obstruction in the mild group had less effect on the inhaled volume during loaded breathing. Therefore, the evolution of $\Delta$ bioZ parameter over different inspiratory loads evidences the volume limitation of severe COPD patients in a noninvasive and comfortable way.

In addition to bioimpedance, we also extracted parameters from myographic signals, $s E M G_{l i c}$, and, $\left|s M M G_{l i c}\right|$. These myographic signals measure the electrical and mechanical activity of the inspiratory muscles of the lower chest wall. As shown in previous studies involving healthy and COPD subjects [9], [18], [38], the higher the imposed inspiratory load the higher the inspiratory muscle activation values.

In this study, $s E M G_{l i c}$ was measured as a noninvasive alternative to crural diaphragm electromyography [18], [19]. The invasively measured crural diaphragm electromyography is used as an estimation of the neural respiratory drive and it is commonly used as a severity marker in respiratory diseases [39]. Fig. 3 shows $f S E s E M G_{\text {lic }}$ for each severity group. Note that during quiet breathing the fSEsEMG $G_{l i c}$ values of the severe group were significantly higher than the values of the mild group. These results agree with Jolley et al. [40] 
who found that higher neural respiratory drive values were related to disease severity being significantly higher compared to healthy subjects during quiet breathing. We also found significant differences between the groups in the $12 \%$, and $24 \%$ loads which confirm the results showed by Duiverman et al. [9] during an inspiratory loaded breathing.

Similar to $f S E s E M G_{l i c}$, we obtained significant differences in $f S E s\left|M M G_{l i c}\right|$ during all the protocol parts as shown in Fig. 3. The $f S E s\left|M M G_{l i c}\right|$ values were higher for the severe patients compared to the mild and moderate ones. Similar results were shown in [21] when comparing COPD patients and healthy subjects during an incremental ventilatory effort. Therefore, severe COPD patients needed significantly higher muscle activity levels, both electrical and mechanical, to breath and overcome the loads, and these differences are reflected in $f S E s E M G_{l i c}$ and $f S E s\left|M M G_{l i c}\right|$.

The myographic parameters used in the presented study, $f S E s E M G_{l i c}$ and $f S E s\left|M M G_{l i c}\right|$ were computed using the fSampEn. This technique has been proposed to estimate the muscle activity from myographic signals because of its capability to reduce the interference from cardiac activity [10], [33].

\section{BEr and $B M r$ ratios}

COPD patients frequently suffer from respiratory muscle dysfunction that contributes to the feeling of breathlessness [24], [25]. To quantify this dysfunction, measurements of inspiratory muscle activity and global respiratory output are commonly compared in order to assess the efficiency of respiratory muscles in ventilation.

Neuro ventilatory coupling evaluates the efficiency of the neural respiratory drive in generating volume and it is commonly computed as the ratio between ventilation and crural diaphragm electromyography. Previous studies have reported neuro ventilatory uncoupling (lower ratio values) in COPD patients during exercise, and showed that this uncoupling is closely related to breathlessness [41]. This kind of examinations require uncomfortable and invasive measurements to be obtained, which is a disadvantage for disease monitoring.

We propose a novel index, BEr, to quantify the contribution of lower chest wall inspiratory muscle activation to ventilation, as the ratio of bioimpedance to surface electromyography measurements. We observed significant differences between the mild and severe COPD groups (Fig. 5). These differences can be also observed in Fig. 4 where these two groups were in discernible zones for all the loads. Specifically, the $\mathrm{BEr}$ median ratio values were higher for the mild than for the severe group during quiet and loaded breathing. These results reflected that the electrical activation of the lower chest wall muscles in severe COPD patients was less efficient in generating impedance changes than in mild COPD patients. Since the relationship between bioimpedance and respiratory volume is essentially linear [6]-[8], [11], the BEr may reflect the efficiency of the neural inspiratory muscle activity in generating respiratory volume.

Our study presents a novel mechanical index, BMr, which included the variation in bioimpedance amplitude with, in this case, the mechanical muscle activity in the lower chest wall $\left(f S E\left|s M M G_{l i c}\right|\right)$. We found significant differences in $\mathrm{BMr}$ between the mild and severe COPD groups during quiet and loaded breathing. Similarly, Sarlabous et al. proposed a ratio between the mouth pressure and the $f S E\left|s M M G_{l i c}\right|$ for COPD patients. Their results showed lower efficiency for the very severe COPD patients than for the severe ones [20]. Our results agree with this previous study, showing that the contribution of mechanical activity of the lower chest muscles to ventilation was lower in severe than in mild COPD patients. The presented BMr ratio used bioimpedance instead of mouth pressure which means less obtrusive measurements compared to [20].

$\mathrm{BEr}$ and BMr behaviors were very similar in this study. However, these indices provide complementary information since the nature of $s E M G_{l i c}$ and $\left|s M M G_{l i c}\right|$ signals is different. The former reflecting the electrical activation and the later the mechanical activation of the lower chest wall inspiratory muscles.

The significant differences in $\mathrm{BEr}$ and $\mathrm{BMr}$ were found between the severity groups which divided the patients by their spirometry results, in particular, by their $\mathrm{FEV}_{1}$. Nevertheless, spirometry and inspiratory threshold loading protocols have different purposes. Spirometry assesses the pulmonary function, and inspiratory loading protocols evaluate the inspiratory muscle activation at different load levels. Consequently, the aim of the presented method was not to assess the pulmonary function as spirometry does. The actual aim was to evaluate the contribution of the muscle activity into global respiratory ventilation by the ratios proposed. On the other hand, inspiratory muscle training can be used in COPD patients with muscle weakness and improves the muscle strength as well as the dyspnea and life quality [42]. Therefore, tracking BEr and $\mathrm{BMr}$ ratios over time may provide useful additional information to the classical assessment in terms of contributions of the inspiratory muscles to ventilation.

\section{Potential use in clinical application}

The main novelty of the present study is the combination of parameters from bioimpedance and myographic signals for respiratory monitoring. To the best of our knowledge, this is the first time evaluating this combination in COPD patients. However, bioimpedance, sEMG and sMMG have been broadly analyzed separately for respiratory purposes. In case of sEMG and sMMG strong correlations between these noninvasive measures and the classical invasive ones [17]-[19]. On the other hand, the relationship between thoracic bioimpedance and respiratory volume was evaluated, showing a clear linear relation [6]-[8], [13]. Other noninvasive techniques have been shown to be able to provide useful respiratory information [12] but, our preference for ventilatory information was thoracic bioimpedance due to its capability to be acquired in a less intrusive wearable system [43], [44]. Consequently, the three noninvasive measures used in this study have been demonstrated to be capable to assess respiration independently. Therefore, our methodological innovation is the combination of bioimpedance and myographic signals as a measure of the 
contribution of inspiratory muscle activity into ventilation in COPD patients.

We acquired bioimpedance and myographic signals using a wearable research device and a wired standard acquisition system, respectively. Nevertheless, nowadays the use of wearable devices to acquire physiological signals is increasing and these devices have already been used in research studies including bioimpedance or myographic signals [44]-[48]. The application of the methods described in the present study requires a multimodal approach to lighten the intrusiveness. In this regard, the acquisition systems are continuously evolving and particularly, the system on chip (SoC) we used [49] currently extended its multimodal feature to fully support the methods we described [50], [51]. The new version of the SoC is included in a new wearable research device and its multimodal capabilities were demonstrated [43]. Consequently, the current versions of the SoC allows bioimpedance, sEMG and sMMG simultaneous recording [50], [51] which reinforces the unobtrusive breathing monitoring and its potential use at home.

Regarding the applicability of the presented study, other key point to consider is the electrodes and sensors location. It is important to maintain approximately the same position to get comparable results over time and the opposite may introduce variability in biompedance measurements as well as in myographic ones [7], [52]. In this way, the use of patches or vests including the sensors [51], [53] will reduce this variability and improve the reproducibility of the measurements.

The assessment of COPD severity includes spirometry and other information sources like questionnaires [4] because of the complex clinical condition of the patients. This current assessment requires the movement of patients to the medical centers. We propose two novel ratios, BEr and BMr, acquired from a single wearable device as noninvasive and portable technique for breathing monitoring. These ratios provide information concerning the relationship of inspiratory muscle activity with global ventilation. The significant differences found in $\mathrm{BEr}$ and $\mathrm{BMr}$ suggest that tracking these ratios provide additional information about COPD condition. As we discussed above, the information these ratios provide differ from the spirometry results which assess the pulmonary function. In consequence, we hypothesize that the progress of the presented ratios over time can contribute to COPD evolution monitoring by unobtrusive methods at home.

\section{Limitations of the study}

The sample size of the presented study was fifty COPD patients and finally, we analyzed the data from 43 patients. The study cohort was large enough to get conclusive results which were confirmed by statistical analysis although the limited size represents a limit in terms of generalization. The mild group size was smaller than the moderate and severe ones and this may reduce the statistical power. However, the conclusive results shown in the present study are useful for knowing the population trend. A broader population could validate the results to get even more generalization.

The selection of fSampEn parameters is an important issue when assessing respiratory muscle effort and consequently, we selected them based on previous studies findings in myographic respiratory signals [18], [31], [34]. The most common embedding dimensions for SampEn calculation are 1 and 2, and no relevant differences between these values were reported in myographic signals [34]. On the other hand, the tolerance parameter influences the performance of fSampEn as it was shown in [31]. Although the current study did not include an analysis of the fSampEn parameters performance, we selected these parameters values according to the good results shown in myographic respiratory signals [18].

The conclusive results from the two novel ratios motivate further studies focused on the physiological analysis.

\section{CONCLUSIONS}

We observed lower contribution of both electrical and mechanical inspiratory muscle activation in ventilation, measured by means of bioimpedance, sEMG and sMMG changes, in severe COPD patients in comparison to mild ones. Furthermore, the differences between groups were persistent during loaded breathing. Bioimpedance and myographic information was combined in two ratios, $\mathrm{BEr}$ and $\mathrm{BMr}$. The novel ratios were obtained in a noninvasive way and in further studies could be obtained with a single wearable device, opening up the way for unobtrusive noninvasive monitoring of breathing in respiratory patients and particularly the assessment of COPD condition.

\section{REFERENCES}

[1] R. A. Pauwels and K. F. Rabe, "Burden and clinical features of chronic obstructive pulmonary disease (COPD)," The Lancet, vol. 364, no. 9434, pp. $613-620,2004$

[2] D. M. Mannino and A. S. Buist, "Global burden of COPD: risk factors, prevalence, and future trends," The Lancet, vol. 370, no. 9589, pp. 765 $-773,2007$.

[3] B. R. Celli, "The importance of spirometry in COPD and asthma: Effect on approach to management," Chest, vol. 117, no. 2, Supplement, pp. $15 \mathrm{~S}-19 \mathrm{~S}, 2000$.

[4] B. R. Celli et al., "Standards for the diagnosis and treatment of patients with COPD: A summary of the ATS/ERS position paper," pp. 932-946, 2004.

[5] M. R. Miller et al., "Standardisation of spirometry," European Respiratory Journal, vol. 26, no. 2, pp. 319-338, 2005.

[6] A. Grenvik et al., "Impedance pneumography: Comparison between chest impedance changes and respiratory volumes in 11 healthy volunteers," Chest, vol. 62, no. 4, pp. $439-443,1972$

[7] V.-P. Seppä et al., "Assessment of pulmonary flow using impedance pneumography," IEEE Transactions on Biomedical Engineering, vol. 57, no. 9, pp. 2277-2285, Sept 2010.

[8] D. Blanco-Almazán et al., "Wearable bioimpedance measurement for respiratory monitoring during inspiratory loading," IEEE Access, vol. 7 , pp. 89487-89496, 2019.

[9] M. L. Duiverman et al., "Reproducibility and responsiveness of a noninvasive emg technique of the respiratory muscles in COPD patients and in healthy subjects," Journal of Applied Physiology, vol. 96, no. 5, pp. 1723-1729, 2004.

[10] L. Estrada et al., "Improvement in Neural Respiratory Drive Estimation From Diaphragm Electromyographic Signals Using Fixed Sample Entropy," IEEE Journal of Biomedical and Health Informatics, vol. 20, no. 2, pp. 476-485, mar 2016.

[11] K. P. Cohen et al., "Comparison of impedance and inductance ventilation sensors on adults during breathing, motion, and simulated airway obstruction," IEEE Transactions on Biomedical Engineering, vol. 44 no. 7, pp. 555-566, July 1997.

[12] M. Folke et al., "Critical review of non-invasive respiratory monitoring in medical care," Medical and Biological Engineering and Computing, vol. 41, no. 4, pp. 377-383, Jul 2003. 
[13] T. Koivumäki et al., "Bioimpedance-based measurement method for simultaneous acquisition of respiratory and cardiac gating signals," Physiological Measurement, vol. 33, no. 8, pp. 1323-1334, aug 2012.

[14] L. P. Malmberg et al., "Measurement of tidal breathing flows in infants using impedance pneumography," European Respiratory Journal, 2016.

[15] V.-P. Seppä et al., "Airway obstruction is associated with reduced variability in specific parts of the tidal breathing flow-volume curve in young children," ERJ Open Research, vol. 5, no. 2, 2019.

[16] J. Gracia-Tabuenca et al., "Tidal breathing flow profiles during sleep in wheezing children measured by impedance pneumography," Respiratory Physiology \& Neurobiology, vol. 271, p. 103312, 2020.

[17] C. C. Reilly et al., "Neural respiratory drive measured during inspiratory threshold loading and acute hypercapnia in healthy individuals," Experimental Physiology, vol. 98, no. 7, pp. 1190-1198, jul 2013.

[18] M. Lozano-García et al., "Surface mechanomyography and electromyography provide non-invasive indices of inspiratory muscle force and activation in healthy subjects," Scientific Reports, vol. 8, no. 1, p. 16921, dec 2018.

[19] L. Lin et al., "Correlation of surface respiratory electromyography with esophageal diaphragm electromyography," Respiratory Physiology \& Neurobiology, vol. 259, pp. $45-52,2019$.

[20] L. Sarlabous et al., "Efficiency of mechanical activation of inspiratory muscles in COPD using sample entropy," European Respiratory Journal, vol. 46, no. 6, 2015.

[21] L. Sarlabous et al., "Inspiratory muscle activation increases with COPD severity as confirmed by non-invasive mechanomyographic analysis," PLOS ONE, vol. 12, no. 5, pp. 1-14, 052017.

[22] M. Lozano-García et al., "Noninvasive assessment of inpiratory muscle neuromechanical coupling during inspiratory threshold loading," IEEE Access, vol. 7, pp. 89487-89496, 2019.

[23] J. C. Hogg, "Pathophysiology of airflow limitation in chronic obstructive pulmonary disease," The Lancet, vol. 364, no. 9435, pp. 709 - 721, 2004.

[24] D. F. Rochester and N. M. T. Braun, "Determinants of maximal inspiratory pressure in chronic obstructive pulmonary disease," American Review of Respiratory Disease, vol. 132, no. 1, pp. 42-47, 1985.

[25] C. A. C. Ottenheijm et al., "Diaphragm dysfunction in chronic obstructive pulmonary disease," American Journal of Respiratory and Critical Care Medicine, vol. 172, no. 2, pp. 200-205, 2005.

[26] D. K. McKenzie et al., "Respiratory muscle function and activation in chronic obstructive pulmonary disease," Journal of Applied Physiology, vol. 107, no. 2, pp. 621-629, 2009.

[27] P. R. Eastwood et al., "Ventilatory responses to inspiratory threshold loading and role of muscle fatigue in task failure." Journal of applied physiology (Bethesda, Md. : 1985), vol. 76, no. 1, pp. 185-95, jan 1994.

[28] F. Laghi et al., "Does resistive loading decrease diaphragmatic contractility before task failure?" Journal of Applied Physiology, vol. 85, no. 3, pp. 1103-1112, sep 1998.

[29] "ATS/ERS statement on respiratory muscle testing," American Journal of Respiratory and Critical Care Medicine, vol. 166, no. 4, pp. 518-624, 2002.

[30] M. Petitjean and F. Bellemare, "Phonomyogram of the diaphragm during unilateral and bilateral phrenic nerve stimulation and changes with fatigue," Muscle \& Nerve, vol. 17, no. 10, pp. 1201-1209, oct 1994.

[31] M. Lozano-García et al., "Performance Evaluation of Fixed Sample Entropy in Myographic Signals for Inspiratory Muscle Activity Estimation," Entropy, vol. 21, no. 2, p. 183, feb 2019.

[32] J. S. Richman and J. R. Moorman, "Physiological time-series analysis using approximate and sample entropy," American Journal of Physiology - Heart and Circulatory Physiology, 2000.

[33] L. Sarlabous et al., "Evidence towards improved estimation of respiratory muscle effort from diaphragm mechanomyographic signals with cardiac vibration interference using sample entropy with fixed tolerance values," PLOS ONE, vol. 9, no. 2, 2014.

[34] L. Estrada et al., "Influence of parameter selection in fixed sample entropy of surface diaphragm electromyography for estimating respiratory activity," Entropy, vol. 19, no. 9, 2017.

[35] J. A. Fiz et al., "Detecting unilateral phrenic paralysis by acoustic respiratory analysis," PLOS ONE, vol. 9, no. 4, pp. 1-9, 042014.

[36] D. Blanco-Almazán et al., "Chest Movement and Respiratory Volume both Contribute to Thoracic Bioimpedance during Loaded Breathing," Scientific Reports, vol. 8, no. -, pp. -, dec 2019.

[37] P. Eastwood et al., "The effects of learning on the ventilatory responses to inspiratory threshold loading," American Journal of Respiratory and Critical Care Medicine, vol. 158, no. 4, pp. 1190-1196, 1998.

[38] M. A. C. Myrrha et al., "Chest wall volumes during inspiratory loaded breathing in COPD patients," Respiratory Physiology \& Neurobiology, vol. 188 , no. 1 , pp. $15-20,2013$.
[39] C. C. Reilly et al., "Neural respiratory drive, pulmonary mechanics and breathlessness in patients with cystic fibrosis," Thorax, vol. 66, no. 3, pp. 240-246, 2011

[40] C. J. Jolley et al., "Neural respiratory drive in healthy subjects and in COPD," European Respiratory Journal, vol. 33, no. 2, pp. 289-297, 2009.

[41] C. J. Jolley et al., "Neural respiratory drive and breathlessness in COPD," European Respiratory Journal, vol. 45, no. 2, pp. 355-364, 2015.

[42] R. Gosselink et al., "Impact of inspiratory muscle training in patients with copd: what is the evidence?" European Respiratory Journal, vol. 37, no. 2, pp. 416-425, 2011

[43] S. Lee et al., "Nightingale v2: Low-power compact-sized multi-sensor platform for wearable health monitoring," in 2019 41st Annual International Conference of the IEEE Engineering in Medicine and Biology Society (EMBC), July 2019, pp. 1290-1293.

[44] T. Van Steenkiste et al., "Portable detection of apnea and hypopnea events using bio-impedance of the chest and deep learning," IEEE Journal of Biomedical and Health Informatics, pp. 1-1, 2020.

[45] A. Burns et al., "Shimmer ${ }^{\mathrm{TM}}$ - a wireless sensor platform for noninvasive biomedical research," IEEE Sensors Journal, vol. 10, no. 9, pp. 15271534, Sep. 2010.

[46] J. Choi et al., "Development and evaluation of an ambulatory stress monitor based on wearable sensors," IEEE Transactions on Information Technology in Biomedicine, vol. 16, no. 2, pp. 279-286, March 2012.

[47] A. Phinyomark et al., "Feature extraction and selection for myoelectric control based on wearable emg sensors," Sensors, vol. 18, no. 5, p. 1615, 2018.

[48] M. Ràfols-de-Urquía et al., "Evaluation of a wearable device to determine cardiorespiratory parameters from surface diaphragm electromyography," IEEE Journal of Biomedical and Health Informatics, vol. 23, no. 5, pp. 1964-1971, Sep. 2019

[49] N. Van Helleputte et al., "A $345 \mu \mathrm{w}$ multi-sensor biomedical soc with bio-impedance, 3-channel ecg, motion artifact reduction, and integrated dsp," IEEE Journal of Solid-State Circuits, vol. 50, no. 1, pp. 230-244, Jan 2015.

[50] M. Konijnenburg et al., "A multi(bio)sensor acquisition system with integrated processor, power management, $8 \times 8$ led drivers, and simultaneously synchronized ecg, bio-z, gsr, and two ppg readouts," IEEE Journal of Solid-State Circuits, vol. 51, no. 11, pp. 2584-2595, Nov 2016.

[51] S. Song et al., "A $769 \mu \mathrm{w}$ battery-powered single-chip soc with ble for multi-modal vital sign monitoring health patches," IEEE Transactions on Biomedical Circuits and Systems, vol. 13, no. 6, pp. 1506-1517, Dec 2019.

[52] L. Mesin et al., "Surface emg: The issue of electrode location," Journal of Electromyography and Kinesiology, vol. 19, no. 5, pp. $719-726$, 2009.

[53] P. Gastelurrutia et al., "Wearable vest for pulmonary congestion tracking and prognosis in heart failure: A pilot study," International journal of cardiology, vol. 215, pp. 77-79, 2016. 